\title{
Correction to: Geometric Reid's recipe for dimer models
}

\author{
Raf Bocklandt ${ }^{1}$ - Alastair Craw ${ }^{2}$ - Alexander Quintero Vélez ${ }^{3}$
}

Published online: 27 January 2021

() Springer-Verlag GmbH Germany, part of Springer Nature 2021

\section{Correction to: Mathematische Annalen https://doi.org/10.1007/s00208-014-1085-8}

The main results of [1], especially Theorems 1.1, 1.4 and Corollary 1.2, are correct as written. However, the final sentence in the statement of Proposition 1.3 is false when the quiver $\mathrm{Q}$ contains a loop at a vertex $i \in \mathrm{Q}_{0}$. When this is the case, there exist points $y \in \mathcal{M}_{\vartheta}$ for which the corresponding $A$-module $V_{y}$ contains a submodule of dimension vector $S_{i}$ that is not isomorphic to $S_{i}$; note that any such $V_{y}$ is not nilpotent. This situation is very rare, ${ }^{1}$ but it does occur.

Example 1 For the action of type $\frac{1}{2}(1,1,0)$, let $y$ be a generic point in the (noncompact) exceptional divisor in $G-\operatorname{Hilb}\left(\mathbb{C}^{3}\right)$, so $y \notin \tau^{-1}\left(x_{0}\right)$. The nonzero maps in the

\footnotetext{
$\overline{{ }^{1} \text { If } \mathrm{Q} \text { has a loop at vertex } i \in \mathrm{Q}_{0}}$, then the locus $\tau^{-1}\left(x_{0}\right)$ is one-dimensional. Indeed, let $n_{1}, \ldots, n_{k} \in N$ be the corners of the polygon $P$ and write $\Pi_{1}, \ldots, \Pi_{k}$ for the corresponding perfect matchings. Let $m \in M$ correspond to the loop $\ell$ in $\mathrm{Q}$ at vertex $i$. After reordering the corner perfect matchings if necessary, there exists $1 \leq l \leq k$ such that $\ell \in \Pi_{j}$ if and only if $1 \leq j \leq l$. Then $\left\langle n_{i}, m\right\rangle=\operatorname{deg}_{\Pi_{i}} \ell=1$ for $1 \leq i \leq l$, whereas $\left\langle n_{j}, m\right\rangle=0$ for $l+1 \leq j \leq k$. Choose a $\mathbb{Z}$-basis of $N$ such that the affine span of $P$ is the plane $\{(x, y, 1) \in N \otimes \mathbb{R} \mid x, y \in \mathbb{R}\}$. If we write $m:=(u, v, w) \in M$ in the dual coordinates, then the polygon $P$ is sandwiched between the parallel lines $u x+v y=-w$ and $u x+v y=-w+1$ in the plane. Thus, $P$ contains no internal lattice points, so $\tau^{-1}\left(x_{0}\right)$ contains no surfaces. The proves the assertion.
}

The original article can be found online at https://doi.org/10.1007/s00208-014-1085-8.

$凶$ Alastair Craw

a.craw@bath.ac.uk

Raf Bocklandt

raf.bocklandt@gmail.com

Alexander Quintero Vélez

aquinte2@unal.edu.co

1 Korteweg de Vries Instituut voor Wiskunde, Universiteit van Amsterdam, P.O. Box 94248, 1090 GE Amsterdam, The Netherlands

2 Department of Mathematical Sciences, University of Bath, Bath BA2 7AY, UK

3 Escuela de Matematicas, Universidad Nacional de Colombia Sede Medellin, Carrera 65 \# 59A-110, Medellin, Colombia 
$A$-module $V_{y}$ are shown

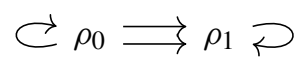

where $\rho_{0}$ and $\rho_{1}$ are the trivial and nontrivial representations of $\mathbb{Z} / 2$ respectively. The submodule $W_{y} \subset V_{y}$ of dimension vector $S_{1}$ destabilises $V_{y}$ as $\vartheta \in C$ moves into the wall $\bar{C} \cap S_{i}^{\perp}$ of the GIT chamber $C$, so $y$ lies in the unstable locus of this wall. However, $W_{y} \nsucceq S_{1}$, so $S_{i} \nsubseteq \operatorname{soc}\left(V_{y}\right)$.

This example shows that even when $\bar{C} \cap S_{i}^{\perp}$ is a wall of the chamber $C$, the unstable locus of the wall need not coincide with $Z_{i}:=\left\{y \in Y \mid S_{i} \subseteq \operatorname{soc}\left(V_{y}\right)\right\}$. In such cases, the final sentence of Proposition 1.3 is false; that sentence should instead conclude that:

...the locus $Z_{i}$ is the intersection of $\tau^{-1}\left(x_{0}\right)$ with

the unstable locus of the wall $\bar{C} \cap S_{i}^{\perp}$.

Indeed, $Z_{i}$ is a subset of the unstable locus of the wall, but this inclusion is equality if and only if the unstable locus is contained in $\tau^{-1}\left(x_{0}\right)$. Instead, for any point $y \in$ $\tau^{-1}\left(x_{0}\right)$ that lies in the unstable locus for the wall $\bar{C} \cap S_{i}^{\perp}$, the destabilising submodule $W_{y} \subset V_{y}$ of dimension vector $S_{i}$ is necessarily isomorphic to $S_{i}$ since $V_{y}$ is nilpotent, giving $y \in Z_{i}$. This proves the equality $(0.1)$.

The error leads to the omission of a case from Lemma 4.8. We now correct that statement:

Lemma 2 (=Lemma 4.8) Every wall of the chamber $C$ that is ofform $\bar{C} \cap S_{i}^{\perp}$ for some nonzero $i \in \mathrm{Q}_{0}$ is either of type 0 , type $\mathrm{I}$, or it is a type III wall with unstable locus $\mathbb{P}^{1} \times \mathbb{C}$. In particular, the support of $H^{0}\left(\Psi\left(S_{i}\right)\right)$ is a single $(-1,-1)$-curve (in type $\left.\mathrm{I}\right)$, a single $(0,-2)$-curve (in type III) or a connected union of compact torus-invariant divisors (in type 0 ).

Proof The only walls that are excluded here are type III walls for which the unstable locus is $\mathbb{F}_{n}$ for some $n \geq 0$. Suppose that one such wall exists. The wall is of the form $\bar{C} \cap S_{i}^{\perp}$, so Proposition 4.7 implies that $\Psi\left(S_{i}\right)=\left.L_{i}^{-1}\right|_{Z_{i}}$. Since $\mathbb{F}_{n} \subseteq \tau^{-1}\left(x_{0}\right)$, the locus $Z_{i}$ coincides with the unstable locus $\mathbb{F}_{n}$, so the support of $\Psi\left(S_{i}\right)$ is of dimension two. To obtain a contradiction, let $\ell \subset Y$ be the fibre of the contraction $\mathbb{F}_{n} \rightarrow \mathbb{P}^{1}$ induced by the wall. For any $z \in \ell$, the sequence

$$
0 \longrightarrow S_{i} \longrightarrow V_{z} \longrightarrow V_{z} / S_{i} \longrightarrow 0
$$

is the $\theta_{0}$-destabilising sequence for $V_{z}$. In particular, the proof of Ishii-Ueda [2, Proposition 11.31] gives that $\Psi\left(S_{i}\right)=\mathcal{O}_{\ell}(-1)$, so the support of $\Psi\left(S_{i}\right)$ has dimension one, a contradiction. The second statement follows from (0.1) above, where in the type III case we compute $Z_{i}$ to be the intersection of $\tau^{-1}\left(x_{0}\right)$ with the unstable locus $\mathbb{P}^{1} \times \mathbb{C}$, i.e. $Z_{i}$ is the torus-invariant $(0,-2)$-curve in $\mathbb{P}^{1} \times \mathbb{C}$. 
The additional case of the type III wall in Lemma 2 should have been analysed in [1, Lemma 4.10, Proposition 4.11]. We now correct those omissions.

Lemma 3 (= Lemma 4.10) Let $\ell$ be a $(-1,-1)$-curve or a $(0,-2)$-curve in $Y$ that arises as the intersection of $\tau^{-1}\left(x_{0}\right)$ with the unstable locus for a wall of the form $\bar{C} \cap S_{i}^{\perp}$ for some nonzero $i \in \mathrm{Q}_{0}$ that is of type I or type III respectively. Then $\left.L_{j}\right|_{\ell} \cong \mathscr{O}_{\ell}$ for all $j \neq i$ and $\left.L_{i}\right|_{\ell} \cong \mathscr{O}_{\ell}(1)$.

Proof The proof from [1, Lemma 4.10] for a $(-1,-1)$-curve applies verbatim for a $(0,-2)$-curve, but the appropriate reference to the work of Ishii-Ueda in this latter case is [2, Lemma 11.32].

Proposition 4 (= Proposition 4.11) Let $i \in \mathrm{Q}_{0}$ be a nonzero vertex. If $H^{0}\left(\Psi\left(S_{i}\right)\right) \neq 0$, then $\left.\Psi\left(S_{i}\right) \cong L_{i}^{-1}\right|_{Z_{i}}$, where $Z_{i}$ is the intersection of $\tau^{-1}\left(x_{0}\right)$ with the unstable locus for the wall $\bar{C} \cap S_{i}^{\perp}$.

Proof The additional case from Lemma 3 shows that the support of $H^{0}\left(\Psi\left(S_{i}\right)\right)$ can be a single $(0,-2)$-curve $\ell_{i}$ equal to the locus $Z_{i}$ for a type III wall $\bar{C} \cap S_{i}^{\perp}$. The proof from [1, Proposition 4.11] in the case where $\ell_{i}$ is a $(-1,-1)$-curve applies verbatim, except that the required isomorphisms $\left.L_{j}\right|_{\ell_{i}} \cong \mathscr{O}_{\ell_{i}}$ for all $j \neq i$ and $\left.L_{i}\right|_{\ell_{i}} \cong \mathscr{O}_{\ell_{i}}(1)$ are obtained from Lemma 3.

The final correction is in [1, Proof of Theorem 1.1], where in describing the case $H^{0}\left(\Psi\left(S_{i}\right)\right) \neq 0$, the locus $Z_{i}$ should equal the intersection of $\tau^{-1}\left(x_{0}\right)$ with the unstable locus of the wall $\bar{C} \cap S_{i}^{\perp}$. In particular, this locus $Z_{i}$ can be either a single $(-1,-1)$-curve, a single $(0,-2)$-curve or a connected union of compact torusinvariant divisors according to the type of the wall as in Lemma 2.

It remains to note that [1, Conjecture 1.5] should refer to the intersection of $\tau^{-1}\left(x_{0}\right)$; in what follows, we take the determinant of $L_{\rho}^{\vee}$ before restricting to $Z_{\rho}$ (this operation was omitted in [1]):

Conjecture 5 (= Conjecture 1.5) The object $\Psi\left(S_{\rho}\right)$ is a pure sheaf in degree 0 if and only if $\bar{C} \cap S_{\rho}^{\perp}$ is a wall of the chamber $C$ defining $G-\mathrm{Hilb}\left(\mathbb{C}^{3}\right)$, in which case $\left.\Psi\left(S_{\rho}\right) \cong \operatorname{det}\left(L_{\rho}^{\vee}\right)\right|_{Z_{\rho}}$ where $Z_{\rho}$ is the intersection of $\tau^{-1}\left(x_{0}\right)$ with the unstable locus of the wall $\bar{C} \cap S_{\rho}^{\perp}$.

\section{References}

1. Bocklandt, R., Craw, A., Vélez, A.Q.: Geometric Reid's recipe for dimer models. Math. Ann. 361(3-4), 689-723 (2015)

2. Ishii, A., Ueda, K.: Dimer models and Crepant resolutions. Hokkaido Math. J. 45(1), 1-42 (2016)

Publisher's Note Springer Nature remains neutral with regard to jurisdictional claims in published maps and institutional affiliations. 